Bull. Korean Math. Soc. 48 (2011), No. 1, pp. 183-195

DOI 10.4134/BKMS.2011.48.1.183

\title{
INHERITED PROPERTIES THROUGH THE HELTON CLASS OF AN OPERATOR
}

\author{
Insook Kim, Yoenha Kim, Eungil Ko, and Ji Eun Lee
}

\begin{abstract}
In this paper we show that Helton class preserves the nilpotent and finite ascent properties. Also, we show some relations on nontransitivity and decomposability between operators and their Helton classes. Finally, we give some applications in the Helton class of weighted shifts.
\end{abstract}

\section{Introduction}

Let $H$ be a complex (separable) Hilbert space and let $\mathcal{L}(H)$ denote the algebra of all bounded linear operators on $H$. A closed linear manifold $\mathcal{M}$ of $H$ is called a nontrivial invariant subspace for an operator $T$ in $\mathcal{L}(H)$ if $T(\mathcal{M}) \subset \mathcal{M}$ and $(0) \neq \mathcal{M} \neq H$. Whether every operator in $\mathcal{L}(H)$ has a nontrivial invariant subspace is an unsolved problem, but some special classes of operators are known to have nontrivial invariant subspaces. We have thought that if an operator $R$ in $\mathcal{L}(H)$ has a nontrivial invariant subspace and $S \in \operatorname{Helton}_{k}(R)$, does $S$ have a nontrivial invariant subspace? Also, what kind of properties of $R$ do Helton $_{k}(R)$ preserve? In this paper we show that Helton class preserves the nilpotent and finite ascent properties. Also, we show some relations on nontransitivity and decomposability between operators and their Helton classes. Finally, we give some applications in the Helton class of weighted shifts.

If $T \in \mathcal{L}(H)$, we write $\sigma(T), \sigma_{p}(T), \sigma_{a p}(T), \sigma_{\text {surj }}(T), \sigma_{l}(T)$, and $\sigma_{r}(T)$ for the spectrum, point spectrum, approximate point spectrum, surjectivity spectrum, left spectrum, and right spectrum of $T$, respectively. Let $\mathcal{K}(H)$ denote the set of compact operators on $H$. We consider the natural map $\pi: \mathcal{L}(H) \rightarrow \mathcal{L}(H) / \mathcal{K}(H)$. Then $\mathcal{L}(H) / \mathcal{K}(H)$ is a Calkin algebra. If $T \in \mathcal{L}(H)$, then the essential spectrum of $T, \sigma_{e}(T)$, is the spectrum of $\pi(T)$ in $\mathcal{L}(H) / \mathcal{K}(H)$ (i.e., $\left.\sigma_{e}(T)=\sigma(\pi(T))\right)$. Similarly, the left and right essential spectra of $T$ are defined by $\sigma_{l e}(T)=\sigma_{l}(\pi(T))$ and $\sigma_{r e}(T)=\sigma_{r}(\pi(T))$, respectively.

Received June 17, 2009.

2010 Mathematics Subject Classification. Primary 47B20; Secondary 47A10.

Key words and phrases. Helton class, nilpotent property, finite ascent property, essential spectra, decomposability.

This work is supported by the Korea Research Foundation Grant funded by Korean Government (MOEHRD, Basic Research Promotion Fund)(KRF-2008-314-C00016). 
An operator $T$ is said to be left semi-Fredholm if there is an operator $R \in$ $\mathcal{L}(H)$ and a compact operator $K$ on $H$ such that $R T=I+K$. An operator $T$ is said to be right semi-Fredholm if there is an operator $R \in \mathcal{L}(H)$ and a compact operator $K^{\prime}$ on $H$ such that $T R=I+K^{\prime}$. An operator $T \in \mathcal{L}(H)$ is hyponormal if $T^{*} T \geq T T^{*}$.

An operator $T \in \mathcal{L}(H)$ is said to have the single valued extension property if for any analytic function $f: D \rightarrow H, D \subset \mathbb{C}$ open, with $(\lambda-T) f(\lambda) \equiv 0$, it results $f(\lambda) \equiv 0$. For an operator $T \in \mathcal{L}(H)$ having the single valued extension property and for $x \in H$ we can consider the set $\rho_{T}(x)$ of elements $\lambda_{0} \in \mathbb{C}$ such that there exists an analytic function $f(\lambda)$ defined in a neighborhood of $\lambda_{0}$, with values in $H$, which satisfies $(\lambda-T) f(\lambda) \equiv x$. Throughout this paper, we denote $\sigma_{T}(x)=\mathbb{C} \backslash \rho_{T}(x)$ and $H_{T}(F)=\left\{x \in H: \sigma_{T}(x) \subset F\right\}$, where $F$ is a subset of $\mathbb{C}$.

Let $R$ and $S$ be in $\mathcal{L}(H)$ and let $C(R, S): \mathcal{L}(H) \rightarrow \mathcal{L}(H)$ be defined by $C(R, S)(A)=R A-A S$. Then

$$
C(R, S)^{k}(I)=\sum_{j=0}^{k}(-1)^{k-j}\left(\begin{array}{c}
k \\
j
\end{array}\right) R^{j} S^{k-j} .
$$

For $R \in \mathcal{L}(H)$ if there is an integer $k \geq 1$ such that an operator $S$ satisfies $C(R, S)^{k}(I)=0$, we say that $S$ belongs to Helton class of $R$ with order $k$. We denote this by $S \in \operatorname{Helton}_{k}(R)$.

\section{Some properties}

In this section we discuss some properties of Helton class of an operator. First, we begin with the nilpotent property.

Theorem 2.1. Let $R \in \mathcal{L}(H)$ be a nilpotent operator of order $m$. If $S \in$ $\operatorname{Helton}_{k}(R)$, then $S$ is a nilpotent operator of order $k+m-1$.

Proof. Since $S \in \operatorname{Helton}_{k}(R)$, it follows that

$$
\sum_{j=0}^{k}(-1)^{k-j}\left(\begin{array}{c}
k \\
j
\end{array}\right) R^{j} S^{k-j}=0 .
$$

Claim. $R^{j} S^{(k+m-1)-j}=0$ for $j=1,2, \ldots, m-1$.

We prove this claim by induction. If $j=m-1$, multiply both sides of (2) by $R^{m-1}$. Then, we get that

$$
\sum_{j=0}^{k}(-1)^{k-j}\left(\begin{array}{l}
k \\
j
\end{array}\right) R^{j+(m-1)} S^{k-j}=0 .
$$

Since $R^{m}=0$, we obtain $R^{m-1} S^{k}=0$. Assume that the claim is true when $j=r+1, r+2, \ldots, m-1$, i.e., $R^{j} S^{(k+m-1)-j}=0$ for $j=r+1, r+2, \ldots, m-1$. 
Then it suffices to show that $R^{r} S^{(k+m-1)-r}=0$. Multiply both sides of (2) by $R^{r}$. Then

$$
\sum_{j=0}^{k}(-1)^{k-j}\left(\begin{array}{c}
k \\
j
\end{array}\right) R^{j+r} S^{k-j}=0 .
$$

Multiply both sides of (4) by $S^{m-(r+1)}$. Then we have

$$
\sum_{j=0}^{k}(-1)^{k-j}\left(\begin{array}{c}
k \\
j
\end{array}\right) R^{j+r} S^{m+k-j-(r+1)}=0 .
$$

By the induction assumption and $(5), R^{r} S^{(k+m-1)-r}=0$. So we complete the proof of the claim.

Since $R^{j} S^{(k+m-1)-j}=0$ for $j=1,2, \ldots, m-1$ by the claim and $R^{m}=0$, we have

$$
\begin{aligned}
0 & =\sum_{j=1}^{k}(-1)^{k-j}\left(\begin{array}{c}
k \\
j
\end{array}\right) R^{j} S^{(k+m-1)-j} \\
& =\left[\sum_{j=1}^{k}(-1)^{k-j}\left(\begin{array}{c}
k \\
j
\end{array}\right) R^{j} S^{k-j}\right] S^{m-1} \\
& =\left[(-1)^{k+1}\left(\begin{array}{c}
k \\
0
\end{array}\right) S^{k}\right] S^{m-1} \\
& =(-1)^{k+1} S^{k+m-1} .
\end{aligned}
$$

Hence $S^{k+m-1}=0$. So we complete the proof.

Recall that an operator $T \in \mathcal{L}(H)$ has finite ascent $m$ if there is some positive integer $m$ such that $\operatorname{ker} T^{m}=\operatorname{ker} T^{m+1}$.

Lemma 2.2. Let $S \in \operatorname{Helton}_{k}(R)$ and $R$ have finite ascent $m$ for some positive integer $m$. If $x \in \operatorname{ker} S^{k+m}$, then $S^{(k+m-1)-j} x \in \operatorname{ker} R^{m}$ for $j=0,1, \ldots, k+$ $m-1$.

Proof. Let $x \in \operatorname{ker} S^{k+m}$. We need to show that $S^{(k+m-1)-j} x \in \operatorname{ker} R^{k+j}$ for $j=0,1, \ldots, k+m-1$. If $j=0$, we multiply both sides of (2) by $S^{k+m-1}$. Then we get that

$$
\left[\sum_{j=0}^{k}(-1)^{k-j}\left(\begin{array}{c}
k \\
j
\end{array}\right) R^{j} S^{k-j}\right] S^{k+m-1}=0 .
$$

Since $S^{k+m} x=0$, we obtain $R^{k} S^{k+m-1} x=0$ and hence $S^{k+m-1} x \in \operatorname{ker} R^{k}$. Assume that $S^{(k+m-1)-j} x \in \operatorname{ker} R^{k+j}$ is true for $j=0,1, \ldots, r-1$, where $r=1,2, \ldots, k+m-1$. Multiply both sides of (2) by $R^{r}$ to get

$$
\sum_{j=0}^{k}(-1)^{k-j}\left(\begin{array}{c}
k \\
j
\end{array}\right) R^{j+r} S^{k-j}=0
$$


and multiply both sides of $(6)$ by $S^{(k+m-1)-r}$. Then we have

$$
\sum_{j=0}^{k}(-1)^{k-j}\left(\begin{array}{c}
k \\
j
\end{array}\right) R^{j+r} S^{2 k+m-1-j-r}=0 .
$$

By the induction assumption and (7), $R^{k+r} S^{(k+m-1)-r} x=0$. Therefore, we have $S^{(k+m-1)-r} x \in \operatorname{ker} R^{k+r}$. Thus $S^{(k+m-1)-(j+1)} x \in \operatorname{ker} R^{k+j+1}$ for $j=$ $0,1, \ldots, r-1$, that is, $S^{(k+m-1)-j} x \in \operatorname{ker} R^{k+j}$ for $j=0,1, \ldots, r$, where $r=$ $0,1, \ldots, k+m-1$. Since $\operatorname{ker} R^{m}=\operatorname{ker} R^{m+1}$, it is easy to show that $\operatorname{ker} R^{k+j}=$ ker $R^{m}$ when $k+j \geq m$. If $k+j<m$, then $\operatorname{ker} R^{k+j} \subset \operatorname{ker} R^{m}$. So we complete the proof.

Theorem 2.3. Let $S \in \operatorname{Helton}_{k}(R)$ and $R$ have finite ascent $m$ for some positive integer $m$. Then $S$ has finite ascent $k+m-1$.

Proof. Let $x \in \operatorname{ker} S^{k+m}$. It suffices to show that $x \in \operatorname{ker} S^{k+m-1}$. If we use the similar method as in the proof of the claim in Theorem 2.1, we can get that $R^{j} S^{(k+m-1)-j} x=0$ for $j=1,2, \ldots, m-1$.

Now multiply both sides of $(2)$ by $S^{m-1}$. Then we get that

$$
\sum_{j=0}^{k}(-1)^{k-j}\left(\begin{array}{c}
k \\
j
\end{array}\right) R^{j} S^{k+m-1-j}=0 .
$$

Then $S^{k+m-1} x=0$ by the claim and Lemma 2.2. So $x \in \operatorname{ker} S^{k+m-1}$ and hence $\operatorname{ker} S^{k+m} \subset \operatorname{ker} S^{k+m-1}$. Therefore, $S$ has finite ascent $k+m-1$.

The theorem above is useful in some sense. In fact, if $R \in \mathcal{L}(H)$ is hyponormal and $S \in \operatorname{Helton}_{k}(R)$, then $S$ has finite ascent $k$ from Theorem 2.3 since $\operatorname{ker} R=\operatorname{ker} R^{2}$.

Corollary 2.4. Let $S \in \operatorname{Helton}_{k}(R)$. If there exists some positive integer $m$ such that $\operatorname{ker}(R-\lambda)^{m}=\operatorname{ker}(R-\lambda)^{m+1}$, then $S$ has the single valued extension property.

Proof. Since $S \in \operatorname{Helton}_{k}(R)$ and the terms of the equation below are equal to zero when $j+s \neq r$, it suffices to consider only the case of $j+s=r$. Then we have the following equations:

$$
\begin{aligned}
& \sum_{j=0}^{k}\left(\begin{array}{c}
k \\
j
\end{array}\right)(-1)^{k-j}(R-\lambda)^{j}(S-\lambda)^{k-j} \\
= & \sum_{j=0}^{k} \sum_{r=0}^{j} \sum_{s=0}^{k-j}(-1)^{k-(s+r)}\left(\begin{array}{c}
k \\
j
\end{array}\right)\left(\begin{array}{c}
j \\
r
\end{array}\right)\left(\begin{array}{c}
k-j \\
s
\end{array}\right) R^{r} \lambda^{j+s-r} S^{k-(j+s)} \\
= & \sum_{j=0}^{k}(-1)^{k-j}\left(\begin{array}{c}
k \\
j
\end{array}\right) R^{j} S^{k-j}=0 .
\end{aligned}
$$


Hence $S-\lambda \in \operatorname{Helton}_{k}(R-\lambda)$ for all $\lambda \in \mathbb{C}$. Since there exists some positive integer $m$ such that $\operatorname{ker}(R-\lambda)^{m}=\operatorname{ker}(R-\lambda)^{m+1}, S-\lambda$ has finite ascent by Theorem 2.3. So $S$ has the single valued extension property by [13].

Corollary 2.5. Let $S \in \operatorname{Helton}_{k}(R)$ and let $A=\left(\begin{array}{cc}0 & I \\ S & 0\end{array}\right)$. If there exists some positive integer $m$ such that $\operatorname{ker}(R-\lambda)^{m}=\operatorname{ker}(R-\lambda)^{m+1}$, then $A$ has the single valued extension property.

Proof. It follows from Corollary 2.4 that $S$ has the single valued extension property. Since $A^{2}=S \bigoplus S$ has the single valued extension property, $A$ has the single valued extension property by [4].

Lemma 2.6. If $S \in \operatorname{Helton}_{k}(R)$ for some integer $k \geq 2$, then $S^{2} \in \operatorname{Helton}_{k}\left(R^{2}\right)$. Moreover, if $k=2$, then

$$
C\left(R^{n+2}, S^{n+2}\right)^{2}(I)=2 R C\left(R^{n+1}, S^{n+1}\right)^{2}(I) S-R^{2} C\left(R^{n}, S^{n}\right)^{2}(I) S^{2}
$$

for any positive integer $n$.

Proof. Since $S \in \operatorname{Helton}_{k}(R), C(R, S)^{k}(I)=0$. Then for any $i=0,1, \ldots, k$, $R^{i} C(R, S)^{k}(I) S^{k-i}=0$ and hence $\sum_{i=0}^{k}\left(\begin{array}{c}k \\ i\end{array}\right) R^{i} C(R, S)^{k}(I) S^{k-i}=0$. So we get that

$$
\begin{aligned}
0 & =\sum_{i=0}^{k}\left(\begin{array}{l}
k \\
i
\end{array}\right) R^{i}\left[\sum_{j=0}^{k}(-1)^{k-j}\left(\begin{array}{l}
k \\
j
\end{array}\right) R^{j} S^{k-j}\right] S^{k-i} \\
& =\sum_{i=0}^{k} \sum_{j=0}^{k}\left(\begin{array}{l}
k \\
i
\end{array}\right)\left(\begin{array}{l}
k \\
j
\end{array}\right)(-1)^{k-j} R^{i+j} S^{2 k-(i+j)} \\
& =\sum_{l=0}^{2 k} \sum_{j=0}^{l}\left(\begin{array}{l}
k \\
j
\end{array}\right)\left(\begin{array}{c}
k \\
l-j
\end{array}\right)(-1)^{k-j} R^{l} S^{2 k-l},
\end{aligned}
$$

where $\left(\begin{array}{c}k \\ j\end{array}\right)=0$ for $k<j$. Since the terms $\sum_{j=0}^{l}\left(\begin{array}{c}k \\ j\end{array}\right)\left(\begin{array}{c}k \\ l-j\end{array}\right)(-1)^{k-j}$ vanish when $l$ is an even number, the equation above becomes $\sum_{t=0}^{k}(-1)^{k-t}\left(\begin{array}{c}k \\ t\end{array}\right) R^{2 t} S^{2 k-2 t}=0$, which implies $S^{2} \in \operatorname{Helton}_{k}\left(R^{2}\right)$.

Moreover, if $k=2$, then by induction, we can obtain the result.

The following theorem shows that Helton class preserves strongly quasinilpotent property.

Theorem 2.7. Let $S \in \operatorname{Helton}_{2}(R)$. If $\lim _{n \rightarrow \infty}\left\|S^{n} x\right\|^{\frac{1}{n}}=0$ for all $x \in H$, then $\lim _{n \rightarrow \infty}\left\|R^{n} x\right\|^{\frac{1}{n}}=0$ for all $x \in H$.

Proof. Since $S \in \operatorname{Helton}_{2}(R)$, we can get that $S^{n} \in \operatorname{Helton}_{k}\left(R^{n}\right)$ for any positive integer $n$ and any integer $k \geq 2$. Indeed, if $n=2$, we have $S^{2} \in$ $\operatorname{Helton}_{2}\left(R^{2}\right)$ by Lemma 2.6. Assume that $S^{n} \in \operatorname{Helton}_{2}\left(R^{n}\right)$ holds for all integer $n \leq m$. By induction, we want to show that $S^{m+1} \in \operatorname{Helton}_{2}\left(R^{m+1}\right)$. From Lemma 2.6, we get that

$$
C\left(R^{m+1}, S^{m+1}\right)^{2}(I)=2 R C\left(R^{m}, S^{m}\right)^{2}(I) S-R^{2} C\left(R^{m-1}, S^{m-1}\right)^{2}(I) S^{2} .
$$


Since $C\left(R^{m}, S^{m}\right)^{2}(I)=C\left(R^{m-1}, S^{m-1}\right)^{2}(I)=0$ by induction hypotheses,

$$
C\left(R^{m+1}, S^{m+1}\right)^{2}(I)=0 .
$$

Hence $S^{m+1} \in \operatorname{Helton}_{2}\left(R^{m+1}\right)$. Thus $S^{n} \in \operatorname{Helton}_{2}\left(R^{n}\right)$ for any positive integer $n$. Since $\operatorname{Helton}_{2}\left(R^{n}\right) \subset \operatorname{Helton}_{k}\left(R^{n}\right)$ for any positive integer $k \geq 2$, we conclude that $S^{n} \in \operatorname{Helton}_{k}\left(R^{n}\right)$ for any positive integer $n$ and any $k \geq 2$. Hence

$$
\sum_{j=0}^{k}(-1)^{k-j}\left(\begin{array}{c}
k \\
j
\end{array}\right)\left(R^{n}\right)^{j}\left(S^{n}\right)^{k-j}=0 .
$$

Thus for any $x \in H$ we have

$$
\left[\sum_{j=0}^{k-1}(-1)^{k-j}\left(\begin{array}{c}
k \\
j
\end{array}\right)\left(R^{n}\right)^{j}\left(S^{n}\right)^{k-j-1}\right] S^{n} x=-R^{n k} x .
$$

Since $\lim _{n \rightarrow \infty}\left\|S^{n} x\right\|^{\frac{1}{n}}=0$ for all $x \in H$, it follows that $\lim _{n \rightarrow \infty}\left\|R^{n k} x\right\|^{\frac{1}{n}}=0$ for all $x \in H$ and any integer $k \geq 2$. Hence $\lim _{n \rightarrow \infty}\left\|R^{n k} x\right\|^{\frac{1}{n k}}=0$ for all $x \in H$ and any integer $k \geq 2$. Thus $\lim _{m \rightarrow \infty}\left\|R^{m} x\right\|^{\frac{1}{m}}=0$ for all $x \in H$.

Corollary 2.8. Let $S \in \operatorname{Helton}_{2}(R)$. If $\lim _{n \rightarrow \infty}\left\|\left(R^{*}\right)^{n} x\right\|^{\frac{1}{n}}=0$ for all $x \in H$, then $\lim _{n \rightarrow \infty}\left\|\left(S^{*}\right)^{n} x\right\|^{\frac{1}{n}}=0$ for all $x \in H$.

Proof. Since $S \in \operatorname{Helton}_{2}(R)$, it follows that $R^{*} \in \operatorname{Helton}_{2}\left(S^{*}\right)$. If we apply the same method as in the proof of Theorem 2.7, we can prove this corollary.

\section{Spectral properties}

In this section we study some spectral properties of Helton class of operators. In particular, we show that nontransitivity is preserved through its Helton class. The following theorem shows that a nontrivial invariant subspace under some conditions is inherited through the Helton class.

Theorem 3.1. Let $R \in \mathcal{L}(H)$ have the single valued extension property and let $S \in \operatorname{Helton}_{k}(R)$ for some integer $k \geq 2$.

(1) If at least one of the equalities $\sigma(R)=\sigma_{e}(R)=\sigma_{l e}(R)$ fails to hold or $\sigma_{l e}(R) \neq \sigma_{r e}(R)$, then $S$ has a nontrivial invariant subspace.

(2) If $S$ is transitive (i.e., has no nontrivial invariant subspaces), then $\sigma(R)=\sigma_{e}(R)=\sigma_{l e}(R)$ and $\sigma\left(R^{*}\right)=\sigma_{e}\left(R^{*}\right)=\sigma_{r e}\left(R^{*}\right)$.

Proof. (1) Assume that at least one of the equalities $\sigma(R)=\sigma_{e}(R)=\sigma_{l e}(R)$ fails to hold or $\sigma_{l e}(R) \neq \sigma_{r e}(R)$. Since $\sigma_{R}(x) \subset \sigma_{S}(x)$ for all $x \in H$ by Corollary 3.4.5 in [14] and $R$ has the single valued extension property, it follows from [12] and [14] that $S$ has the single valued extension property, $\sigma(R)=$ $\cup\left\{\sigma_{R}(x): x \in H\right\}$, and $\sigma(S)=\cup\left\{\sigma_{S}(x): x \in H\right\}$. Thus we have $\sigma(R) \subset \sigma(S)$. Since $R^{*} \in \operatorname{Helton}_{k}\left(S^{*}\right), \sigma_{S^{*}}(x) \subset \sigma_{R^{*}}(x)$ for all $x \in H$ and hence $\sigma_{\text {surj }}\left(S^{*}\right)=$ $\cup\left\{\sigma_{S^{*}}(x): x \in H\right\} \subset \cup\left\{\sigma_{R^{*}}(x): x \in H\right\}=\sigma_{\text {surj }}\left(R^{*}\right)$ by [14]. Then $\sigma_{l}(S) \subset$ $\sigma_{l}(R)$, because $\sigma_{\text {surj }}\left(T^{*}\right)=\sigma_{a p}(T)=\sigma_{l}(T)$ for any operator $T \in \mathcal{L}(H)$. Since 
$\pi(S) \in \operatorname{Helton}_{k}(\pi(R)), \sigma_{l}(\pi(S)) \subset \sigma_{l}(\pi(R))$, i.e., $\sigma_{l e}(S) \subset \sigma_{l e}(R)$. Since $R^{*} \in \operatorname{Helton}_{k}\left(S^{*}\right)$, we obtain $\sigma_{l e}\left(R^{*}\right) \subset \sigma_{l e}\left(S^{*}\right)$ and hence $\sigma_{r e}(R) \subset \sigma_{r e}(S)$. Therefore,

$$
\sigma_{l e}(S) \subset \sigma_{l e}(R) \subset \sigma_{e}(R) \subset \sigma(R) \subset \sigma(S) .
$$

So we get that $\sigma_{l e}(S) \neq \sigma(S)$. Then $\sigma_{p}(S) \cup \sigma_{p}\left(S^{*}\right) \neq \phi$. Hence $S$ has a nontrivial invariant subspace.

(2) Since $S$ is transitive, $\sigma(S)=\sigma_{e}(S)=\sigma_{l e}(S)=\sigma_{r e}(S)$. Otherwise, $\sigma_{p}(S) \cup \sigma_{p}\left(S^{*}\right) \neq \phi$. Hence $S$ has a nontrivial invariant subspace. Then we have a contradiction. On the other hand, it is known that $\sigma_{l e}(S) \subset \sigma_{l e}(R) \subset$ $\sigma_{e}(R) \subset \sigma(R) \subset \sigma(S)$ from the proof of (1). So we conclude that $\sigma(R)=$ $\sigma_{e}(R)=\sigma_{l e}(R)$. Since $\sigma_{r e}\left(R^{*}\right)=\sigma_{l e}(R)^{*}$, the second assertion follows.

Now we study some relations between a (semi) Fredholm operator and its Helton class. In the following we obtain easy applications of Theorem 3.1.

Corollary 3.2. Let $R \in \mathcal{L}(H)$ and let $S \in \operatorname{Helton}_{k}(R)$. If $R-\lambda$ is left (semi) Fredholm, then $S-\lambda$ is left (semi) Fredholm. Furthermore, if $S-\lambda$ is right (semi) Fredholm, then $R-\lambda$ is right (semi) Fredholm.

Proof. If $R-\lambda$ is left (semi) Fredholm, then $\lambda \notin \sigma_{l e}(R)$. Hence $\lambda \notin \sigma_{l e}(S)$ by the proof of Theorem 3.1. Thus $S-\lambda$ is left (semi) Fredholm. Similarly, we can get the second statement.

Example 3.3. Let $S$ be the unilateral shift and let $W$ be a unilateral weighted shift which has a weight sequence $\left\{\beta_{n}\right\}_{n=0}^{\infty}$. If $W \in \operatorname{Helton}_{k}(S)$, then $\sigma_{l e}(W) \neq$ $\sigma(W)$ and $W$ has a nontrivial invariant subspace. In fact, since we know that $\sigma_{l e}(S)=\sigma_{r e}(S)=\sigma_{e}(S)=\partial \mathbf{D}$, where $\partial \mathbf{D}$ denotes the boundary of the unit disk and $\sigma(S)=\overline{\mathbf{D}}, \sigma_{l e}(S) \neq \sigma(S)$. Hence the result follows from Theorem 3.1.

Recall that a closed linear subspace $\mathcal{Y}$ of $H$ is called a spectral maximal space of $T$ if $\mathcal{Y}$ is invariant to $T$ and if $\mathcal{Z}$ is another closed linear subspace of $H$, invariant to $T$, such that $\sigma\left(\left.T\right|_{\mathcal{Z}}\right) \subset \sigma\left(\left.T\right|_{\mathcal{Y}}\right)$, then $\mathcal{Z} \subset \mathcal{Y}$. An operator $T \in \mathcal{L}(H)$ is called decomposable if for every finite open covering $\left\{G_{i}\right\}_{i=1}^{n}$ of $\sigma(T)$ there exists a system $\left\{\mathcal{M}_{i}\right\}_{i=1}^{n}$ of spectral maximal spaces of $T$ such that $\sigma\left(\left.T\right|_{\mathcal{M}_{i}}\right) \subset G_{i}$ for every $i=1,2, \ldots, n$ and $H=\sum_{i=1}^{n} \mathcal{M}_{i}$.

Now we show that Helton class preserves decomposability under some conditions.

Theorem 3.4. Let $R \in \mathcal{L}(H)$ be decomposable and let $S \in \operatorname{Helton}_{k}(R)$. If $\sigma_{R}(x) \supset \sigma_{S}(x)$ for all nonzero $x \in H, S^{*}$ has the single valued extension property and $\sigma(R)=\sigma_{R}(x)$ for all nonzero $x \in H$, or $R \in \operatorname{Helton}_{k}(S)$, then $S$ is decomposable.

Proof. Assume that $\sigma_{R}(x) \supset \sigma_{S}(x)$ for all nonzero $x \in H$. Since $R$ has the property $(\beta)$, it follows from [6] that $S$ has the property $(\beta)$. Then $\sigma_{R}(x)=$ $\sigma_{S}(x)$ for all $x \in H$ with the proof of Theorem 3.1. Hence $\sigma(R)=\sigma(S)$. Let $\left\{G_{i}\right\}_{i=1}^{n}$ be a finite open cover of $\sigma(S)$. Then $\left\{G_{i}\right\}_{i=1}^{n}$ is a finite open cover of 
$\sigma(R)$. Since $R$ is decomposable, there exists $\left\{Y_{i}\right\}$, a system of spectral maximal spaces of $R$ such that

$$
\left\{\begin{array}{l}
\sigma\left(\left.R\right|_{Y_{i}}\right) \subset G_{i}, \quad i=1,2, \ldots, n \text { and } \\
H=Y_{1}+\cdots+Y_{n} .
\end{array}\right.
$$

Since $R$ is decomposable and $Y_{i}(i=1,2, \ldots, n)$ are spectral maximal spaces of $R$, from [4] we get that

$$
Y_{i}=H_{R}\left(\sigma\left(\left.R\right|_{Y_{i}}\right)\right), \quad i=1,2, \ldots, n .
$$

Since $\sigma_{R}(x)=\sigma_{S}(x)$ for all $x \in H, H_{R}(F)=H_{S}(F)$ for all closed sets $F \subset \mathbb{C}$ by Corollary 3.4.5 in [14]. So we get that

$$
H_{S}\left(\sigma\left(\left.R\right|_{Y_{i}}\right)\right)=H_{R}\left(\sigma\left(\left.R\right|_{Y_{i}}\right)\right), \quad i=1,2, \ldots, n .
$$

Since $S$ has the property $(\beta)$, from Proposition 1.3.8 in [4] we get that $H_{S}\left(\sigma\left(\left.R\right|_{Y_{i}}\right)\right)$ are spectral maximal spaces of $S$ and

$$
\begin{aligned}
\sigma\left(\left.S\right|_{H_{S}\left(\sigma\left(\left.R\right|_{Y_{i}}\right)\right)}\right) & \subset \sigma(S) \cap \sigma\left(\left.R\right|_{Y_{i}}\right) \\
& \subset \sigma\left(\left.R\right|_{Y_{i}}\right) \subset G_{i} .
\end{aligned}
$$

Since

$$
\begin{aligned}
H_{S}\left(\sigma\left(\left.R\right|_{Y_{1}}\right)\right)+\cdots+H_{S}\left(\sigma\left(\left.R\right|_{Y_{n}}\right)\right) & =H_{R}\left(\sigma\left(\left.R\right|_{Y_{1}}\right)\right)+\cdots+H_{R}\left(\sigma\left(\left.R\right|_{Y_{n}}\right)\right) \\
& =Y_{1}+\cdots+Y_{n}=H
\end{aligned}
$$

we conclude that $S$ is decomposable.

Assume that $S^{*}$ has the single valued extension property and $\sigma(R)=\sigma_{R}(x)$ for all nonzero $x \in H$. Since $R^{*} \in \operatorname{Helton}_{k}\left(S^{*}\right), R^{*}$ has the single valued extension property and hence $\sigma\left(S^{*}\right) \subset \sigma\left(R^{*}\right)$ as in the proof of Theorem 3.1. So we have $\sigma(S) \subset \sigma(R)$. Since $R$ has the single valued extension property from [4], it follows from [12] that $S$ has the single valued extension property. Thus $\sigma(S)=\cup\left\{\sigma_{S}(x): x \in H\right\}$ by [14]. Since $\sigma(R)=\sigma_{R}(x)$ for all nonzero $x \in H$, we get that $\sigma_{R}(x) \supset \sigma_{S}(x)$ for all nonzero $x \in H$ and the argument above asserts that $S$ is decomposable.

In the case of $S \in \operatorname{Helton}_{k}(R)$ and $R \in \operatorname{Helton}_{k}(S)$, the result is obvious.

Recall that an operator $R \in \mathcal{L}(H)$ is said to be a spectral operator (in the sense of Dunford) if $R=T+Q$, where $T$ is a scalar type operator and $Q$ is a quasinilpotent operator commuting with $T$.

Corollary 3.5. Let $R \in \mathcal{L}(H)$ be a spectral operator and let $S \in \operatorname{Helton}_{k}(R)$. If $\sigma_{R}(x) \supset \sigma_{S}(x)$ for all nonzero $x \in H$, then $S$ is decomposable. Furthermore, if $\sigma(S)$ is not reduced to a single point, then $S$ has nontrivial hyperinvariant subspaces in $H$.

Proof. Since $R$ is decomposable from [4], the proof follows from Theorem 3.4. Furthermore, since $S$ is decomposable, $S$ has nontrivial spectral maximal spaces from Theorem 2.1.5 in [4]. Hence Proposition 1.3.2 in [4] implies that $S$ has nontrivial hyperinvariant subspaces in $H$. 
Recall that an operator $R \in \mathcal{L}(H)$ is said to be algebraic if $p(R)=0$ for some nonzero polynomial $p$.

Proposition 3.6. Let $R \in \mathcal{L}(H)$ have the single valued extension property and let $S \in \operatorname{Helton}_{k}(R)$. If $S$ is algebraic, then $R$ is decomposable.

Proof. If $S$ is algebraic, then $p(S)=0$ for some nonzero polynomial $p$. Since $p(\sigma(S))=\sigma(p(S))=\{0\}$ by the spectral mapping theorem, $\sigma(S)$ is contained in the set of zeros of $p$. Since $\sigma(R) \subset \sigma(S)$ as in the proof of Theorem 3.1, $\sigma(R)$ is contained in the set of zeros of $p$. Hence $\sigma(R)$ is a finite set. Hence $R$ is decomposable.

Recall that an operator $T$ is said to satisfy the Dunford's property $(C)$ if the linear manifold $H_{T}(F)$ is closed for each closed subset $F$ of $\mathbb{C}$.

Theorem 3.7. Let $R \in \mathcal{L}(H)$ have the Dunford's property $(C)$ and let $S \in$ $\operatorname{Helton}_{k}(R)$. If $\sigma_{R}(x) \supset \sigma_{S}(x)$ for all nonzero $x \in H$, then $S$ has the Dunford's property $(C)$.

Proof. We have $\sigma_{S}(x)=\sigma_{R}(x)$ for all $x \in H$ by the proof of Theorem 3.4. So $H_{R}(F)=H_{S}(F)$ for each closed subset $F$ of $\mathbb{C}$ by Corollary 3.4.5 in [14]. Since $R$ has the Dunford's property $(C), H_{R}(F)$ is closed for each closed subset $F$ of $\mathbb{C}$. Thus $H_{S}(F)$ is also closed for each closed subset $F$ of $\mathbb{C}$ and hence $S$ has the Dunford's property $(C)$.

Corollary 3.8. Let $R \in \mathcal{L}(H)$ satisfy $\sigma_{R}(x)=\sigma(R)$ for all nonzero $x \in H$ and let $S \in \operatorname{Helton}_{k}(R)$. If $S^{*}$ has the single valued extension property, then $S$ satisfies $\sigma_{S}(x)=\sigma(S)$ for all nonzero $x \in H$ and hence $S$ has the Dunford's property $(C)$.

Proof. Since $\sigma_{R}(x)=\sigma(R)$ for all nonzero $x \in H, H_{R}(F)=\{0\}$ for every closed set $F \subset \mathbb{C}$ that does not contain $\sigma(R)$, while $H_{R}(F)=H$ otherwise. Hence $R$ has the Dunford's property $(C)$ and the single valued extension property. Since $S \in \operatorname{Helton}_{k}(R), \sigma(R) \subset \sigma(S)$ and $\sigma_{R}(x) \subset \sigma_{S}(x)$ for all $x \in H$. If $S \in \operatorname{Helton}_{k}(R)$, it is clear that $R^{*} \in \operatorname{Helton}_{k}\left(S^{*}\right)$. If $S^{*}$ has the single valued extension property, then from [12] we can get that $R^{*}$ has the single valued extension property. Thus $\sigma\left(S^{*}\right) \subset \sigma\left(R^{*}\right)=\sigma(R)^{*}=\sigma_{R}(x)^{*} \subset \sigma_{S}(x)^{*}$ for all nonzero $x \in H$. Hence $\sigma(S) \subset \sigma_{S}(x)$ for all nonzero $x \in H$. So we have $\sigma(S)=\sigma_{S}(x)$ for all nonzero $x \in H$ which implies that $S$ has the Dunford's property $(C)$ as in the argument above.

\section{Some applications in weighted shifts}

In this section we consider the Helton class of weighted shifts. In particular, we study hyponormality and Aluthge transform of an operator between a weighted shift and its Helton class with order two.

Let $\left\{e_{n}\right\}_{n=0}^{\infty}$ be an orthonormal basis of $H$ and let $S$ be the unilateral shift defined by $S e_{n}=e_{n+1}$ for every nonnegative integer $n$. If $\left\{\alpha_{n}\right\}_{n=0}^{\infty}$ is any 
bounded sequence of nonnegative numbers, a unilateral weighted shift $W$ with a weight sequence $\left\{\alpha_{n}\right\}$ is defined by $W e_{n}=\alpha_{n} e_{n+1}$ for every nonnegative integer $n$. We begin with some elementary lemma.

Lemma 4.1. Let $R$ and $W$ be unilateral weighted shifts with weight sequences $\left\{\alpha_{n}\right\}_{n=0}^{\infty}$ and $\left\{\beta_{n}\right\}_{n=0}^{\infty}$, respectively. Then $W \in \operatorname{Helton}_{k}(R)$ if and only if

$$
\begin{gathered}
\sum_{j=1}^{k-1}(-1)^{k-j}\left(\begin{array}{c}
k \\
j
\end{array}\right) \beta_{n} \cdots \beta_{n+k-j-1} \alpha_{n+k-j} \cdots \cdots \alpha_{n+k-1} \\
+(-1)^{k} \beta_{n} \cdots \beta_{n+k-1}+\alpha_{n} \cdots \alpha_{n+k-1}=0
\end{gathered}
$$

for all nonnegative integer $n$.

Theorem 4.2. Let $R$ and $W$ be unilateral weighted shifts with positive weight sequences $\left\{\alpha_{n}\right\}_{n=0}^{\infty}$ and $\left\{\beta_{n}\right\}_{n=0}^{\infty}$, respectively and let $W \in \operatorname{Helton}_{2}(R)$. Then the following statements hold;

(1) $\frac{\beta_{n+1}}{\beta_{n}} \leq \frac{\alpha_{n+1}}{\alpha_{n}}$ for all $n \geq 0$ and $\alpha_{0} \leq \beta_{0}$. In particular, if $W$ is hyponormal, then $R$ is hyponormal.

(2) If $R$ is hyponormal and $\left\{\beta_{n}\right\}_{n=0}^{\infty}$ is decreasing, then

$$
\alpha_{n+1} \geq \beta_{n}+\sqrt{\beta_{n}^{2}-\beta_{n} \beta_{n+1}} \text { or } \alpha_{n+1} \leq \beta_{n}-\sqrt{\beta_{n}^{2}-\beta_{n} \beta_{n+1}} \text {. }
$$

Proof. (1) Since $W \in \operatorname{Helton}_{2}(R)$, from Lemma 4.1 (with $k=2$ ) we have

$$
\beta_{n} \beta_{n+1}-2 \beta_{n} \alpha_{n+1}+\alpha_{n} \alpha_{n+1}=0
$$

for every nonnegative integer $n$. Then

$$
\frac{\beta_{n}}{\alpha_{n}} \frac{\beta_{n+1}}{\alpha_{n+1}}-2 \frac{\beta_{n}}{\alpha_{n}}+1=0 \text { for all } n \geq 0 .
$$

Let $A_{n}=\frac{\beta_{n}}{\alpha_{n}}$. Then the equation above becomes $A_{n} A_{n+1}-2 A_{n}+1=0$, and the sequence $\left\{A_{n}\right\}$ is decreasing, that is, $\frac{\beta_{n+1}}{\alpha_{n+1}} \leq \frac{\beta_{n}}{\alpha_{n}}$. Therefore, $\frac{\beta_{n+1}}{\beta_{n}} \leq \frac{\alpha_{n+1}}{\alpha_{n}}$ for all $n \geq 0$.

We note that $A_{n}=\frac{(n+1) A_{0}-n}{n A_{0}-(n-1)}$ for all $n \geq 1$. This equation is easily obtained by induction. Since $A_{n}>0, A_{0}>\frac{n}{n+1}$ for all $n \geq 1$ by an easy induction, and hence $A_{0} \geq 1$. Thus we have $\alpha_{0} \leq \beta_{0}$.

In particular, if $W$ is hyponormal, then $\frac{\beta_{n+1}}{\beta_{n}} \geq 1$. So $\frac{\alpha_{n+1}}{\alpha_{n}} \geq 1$, which implies that $R$ is hyponormal.

(2) Since $W \in \operatorname{Helton}_{2}(R), W^{2}-2 R W+R^{2}=0$. Then for every nonnegative integer $n$, we have

$$
\beta_{n} \beta_{n+1}-2 \beta_{n} \alpha_{n+1}+\alpha_{n} \alpha_{n+1}=0 .
$$

Hence $\alpha_{n}=\frac{2 \beta_{n} \alpha_{n+1}-\beta_{n} \beta_{n+1}}{\alpha_{n+1}}$. Since $R$ is hyponormal, $\alpha_{n} \leq \alpha_{n+1}$ for every nonnegative integer $n$. Hence

$$
0<\alpha_{n}=\frac{2 \beta_{n} \alpha_{n+1}-\beta_{n} \beta_{n+1}}{\alpha_{n+1}} \leq \alpha_{n+1}
$$


Then $\alpha_{n+1}^{2}-2 \beta_{n} \alpha_{n+1}+\beta_{n} \beta_{n+1} \geq 0$. If we solve the equation $\alpha_{n+1}^{2}-2 \beta_{n} \alpha_{n+1}+$ $\beta_{n} \beta_{n+1}=0$, then we get

$$
\alpha_{n+1}=\beta_{n} \pm \sqrt{\beta_{n}^{2}-\beta_{n} \beta_{n+1}} .
$$

Since $\beta_{n} \geq \beta_{n+1}$ for every nonnegative integer $n, \alpha_{n+1} \geq \beta_{n}+\sqrt{\beta_{n}^{2}-\beta_{n} \beta_{n+1}}$ or $\alpha_{n+1} \leq \beta_{n}-\sqrt{\beta_{n}^{2}-\beta_{n} \beta_{n+1}}$.

Corollary 4.3. Let $S$ be the unilateral shift and $W$ a unilateral weighted shift which has a positive weight sequence $\left\{\beta_{n}\right\}_{n=0}^{\infty}$. If $W \in \operatorname{Helton}_{2}(S)$, then $\left\{\beta_{n}\right\}_{n=0}^{\infty}$ is decreasing with $\beta_{0} \geq 1$.

Proof. It is clear from Theorem 4.2.

Example 4.4. Let $W \in \operatorname{Helton}_{2}(R)$, where $R$ and $W$ are unilateral weighted shifts with positive weight sequences $\left\{\alpha_{n}\right\}_{n=0}^{\infty}$ and $\left\{\beta_{n}\right\}_{n=0}^{\infty}$, respectively. Let $\left\{\beta_{n}\right\}_{n=0}^{\infty}=\left\{\frac{n+1}{n+2}\right\}_{n=0}^{\infty}$. Then $R$ is hyponormal by Theorem 4.2 , since $W$ is hyponormal. Moreover, we can find a weight sequence $\left\{\alpha_{n}\right\}_{n=0}^{\infty}$ of $R$, which is $\alpha_{n}=\frac{n(n+1)-2(n-1)(n+1) \alpha_{0}}{(n+1)(n+2)-2 n(n+2) \alpha_{0}}$, where $\alpha_{0}$ is any positive number with $\alpha_{0} \leq \frac{1}{2}$.

Corollary 4.5. Let $R$ and $W$ be different unilateral weighted shifts with positive weight sequences $\left\{\alpha_{n}\right\}_{n=0}^{\infty}$ and $\left\{\beta_{n}\right\}_{n=0}^{\infty}$, respectively and let $W \in \operatorname{Helton}_{2}(R)$. Suppose that $R$ is hyponormal. If $\left\{\beta_{n}\right\}_{n=0}^{\infty}$ is decreasing, then $\|R\| \geq \beta_{n}$ or $\alpha_{n} \leq\|W\|=\beta_{1}$.

Proof. For every nonnegative integer $n$, Theorem 4.2 implies that $\alpha_{n+1} \geq \beta_{n}$ or $\alpha_{n+1} \leq \beta_{n}$. Since $\|R\|=\sup _{n}\left|\alpha_{n}\right|$, we complete our proof.

For an operator $T \in \mathcal{L}(H)$, let $T=U|T|$ be the polar decomposition of $T$. Then the Aluthge transform of $T$ is defined by $\widetilde{T}=|T|^{\frac{1}{2}} U|T|^{\frac{1}{2}}$ (For more details, see $[10,11])$. If $R$ is a weighted shift with a weight sequence $\left\{\alpha_{n}\right\}_{n=0}^{\infty}$, then the Aluthge transform $\widetilde{R}$ of $R$ is given by $\widetilde{R} e_{n}=\sqrt{\alpha_{n} \alpha_{n+1}} e_{n+1}$ for every nonnegative integer $n$. In the following proposition we show that Helton class does not preserve the Aluthge transform in general.

Proposition 4.6. Let $R$ and $W$ be unilateral weighted shifts with positive weight sequences $\left\{\alpha_{n}\right\}_{n=0}^{\infty}$ and $\left\{\beta_{n}\right\}_{n=0}^{\infty}$, respectively and let $W \in \operatorname{Helton}_{2}(R)$. If $\widetilde{W} \in \operatorname{Helton}_{2}(\widetilde{R})$, then $W=R$.

Proof. Since $W \in \operatorname{Helton}_{2}(R)$ and $\widetilde{W} \in \operatorname{Helton}_{2}(\widetilde{R})$, for every nonnegative integer $n$ we have

$$
\beta_{n} \beta_{n+1}-2 \beta_{n} \alpha_{n+1}+\alpha_{n} \alpha_{n+1}=0
$$

$$
\sqrt{\beta_{n} \beta_{n+1}} \sqrt{\beta_{n+1} \beta_{n+2}}-2 \sqrt{\beta_{n} \beta_{n+1}} \sqrt{\alpha_{n+1} \alpha_{n+2}}+\sqrt{\alpha_{n} \alpha_{n+1}} \sqrt{\alpha_{n+1} \alpha_{n+2}}=0 .
$$

Let $A_{n}=\frac{\beta_{n}}{\alpha_{n}}$. Then the equations (10) and (11) become

$$
A_{n} A_{n+1}-2 A_{n}+1=0
$$


and

$$
\sqrt{A_{n} A_{n+1}} \sqrt{A_{n+1} A_{n+2}}-2 \sqrt{A_{n} A_{n+1}}+1=0 .
$$

We combine the equations (12) and (13) to obtain $A_{n}=A_{n+1}$ for every nonnegative integer $n$, and from (12) we get that $A_{n}=1$ for every nonnegative integer $n$. This implies $\beta_{n}=\alpha_{n}$ for every nonnegative integer $n$. Thus we have $W=R$.

Example 4.7. Let $R$ be the unilateral shift and $W$ a unilateral weighted shift with weight $\left\{\beta_{n}\right\}_{n=0}^{\infty}=\left\{\frac{n+2}{n+1}\right\}_{n=0}^{\infty}$. Then $W \in \operatorname{Helton}_{2}(R)$. But $\widetilde{W} \notin$ $\operatorname{Helton}_{2}(\widetilde{R})$. Indeed, since $\widetilde{W}$ is the unilateral weighted shift with weight sequence $\left\{\sqrt{\frac{n+3}{n+1}}\right\}_{n=0}^{\infty}$ and $\widetilde{R}=R, \sqrt{\frac{n+3}{n+1}} \sqrt{\frac{n+4}{n+2}}-2 \sqrt{\frac{n+3}{n+1}}+1 \neq 0$ for every nonnegative integer $n$.

\section{References}

[1] P. Aiena, Fredholm and Local Spectral Theory, with Applications to Multipliers, Kluwer Academic Publishers, Dordrecht, 2004.

[2] C. Benhida and E. Zerouali, Local spectral theory of linear operators $R S$ and SR, Integral Equations Operator Theory 54 (2006), no. 1, 1-8.

[3] E. Bishop, A duality theorem for an arbitrary operator, Pacific J. Math. 9 (1959), 379397.

[4] I. Colojoară and C. Foiaş, Theory of Generalized Spectral Operators, Gordon and Breach, Science Publishers, New York-London-Paris, 1968.

[5] J. B. Conway, A Course in Functional Analysis, Springer-Verlag, 1985.

[6] B. P. Duggal, Upper triangular operator matrices with the single-valued extension property, J. Math. Anal. Appl. 349 (2009), no. 1, 85-89.

[7] B. P. Duggal and S. V. Djordjević, Dunford's property $(C)$ and Weyl's theorems, Integral Equations Operator Theory 43 (2002), no. 3, 290-297.

[8] J. Eschmeier, Invariant subspaces for subscalar operators, Arch. Math. (Basel) 52 (1989), no. 6, 562-570.

[9] J. Eschmeier and M. Putinar, Bishop's condition $(\beta)$ and rich extensions of linear operators, Indiana Univ. Math. J. 37 (1988), no. 2, 325-348.

[10] I. B. Jung, E. Ko, and C. Pearcy, Aluthge transforms of operators, Integral Equations Operator Theory 37 (2000), no. 4, 437-448.

[11] _ Spectral pictures of Aluthge transforms of operators, Integral Equations Operator Theory 40 (2001), no. 1, 52-60.

[12] Y. Kim, E. Ko, and J. Lee, Operators with the single valued extension property, Bull. Korean Math. Soc. 43 (2006), no. 3, 509-517.

[13] K. Laursen, Operators with finite ascent, Pacific J. Math. 152 (1992), no. 2, 323-336.

[14] K. Laursen and M. Neumann, An Introduction to Local Spectral Theory, The Clarendon Press, Oxford University Press, New York, 2000.

[15] C. Lin, Z. Yan, and Y. Ruan, Common properties of operators $R S$ and SR and phyponormal operators, Integral Equations Operator Theory 43 (2002), no. 3, 313-325.

INSOOK KIM

Department of Mathematics

EWHA WOMEN'S UNIVERSITY

SEOUl 120-750, KorEA

E-mail address: ikim99@hanmail.net 
INHERITED PROPERTIES THROUGH THE HELTON CLASS OF AN OPERATOR 195

\section{Yoenha Kim}

Department of Mathematics

Ewha WoMen's University

SeOul 120-750, Korea

E-mail address: yoenha@ewhain.net

\section{EungIL Ko}

DePARTMENT OF Mathematics

EWha Women's University

SEOUl 120-750, Korea

E-mail address: eiko@ewha.ac.kr

Ji EUN LEE

Institute of Mathematical Sciences

EWha WOMEN's UnIVERSiTy

SeOul 120-750, Korea

E-mail address: jieun7@ewhain.net 